\title{
Primary pleomorphic rhabdomyosarcoma of the adrenal gland in an adult: $A$ case report
}

\author{
CHAO-JUN WANG ${ }^{1}$, JUN LI ${ }^{2}$ and JIE QIN ${ }^{1}$ \\ Departments of ${ }^{1}$ Urology and ${ }^{2}$ Pathology, The First Affiliated Hospital, College of Medicine, \\ Zhejiang University, Hangzhou, Zhejiang 310003, P.R. China
}

Received April 10, 2013; Accepted October 22, 2013

DOI: $10.3892 / \mathrm{ol} .2013 .1674$

\begin{abstract}
A 61-year-old female was referred to The First Affiliated Hospital, College of Medicine, Zhejiang University (Hangzhou, China) due to a right adrenal tumor. A pre-operative transcutaneous fine-needle aspiration biopsy and right adrenalectomy were performed, and pathological analysis resulted in the diagnosis of pleomorphic rhabdomyosarcoma (RMS). Primary pleomorphic RMS of the adrenal gland in an adult is a rare condition. To the best of our knowledge, this is the first case of pleomorphic RMS of the adrenal gland in an adult diagnosed by light microscopy and immunohistochemical stains.
\end{abstract}

\section{Introduction}

Rhabdomyosarcoma (RMS) is a malignant soft-tissue sarcoma that is believed to develop from primitive totipotent embryonic mesenchyme. RMS is a highly aggressive tumor with a tendency for advanced and disseminated disease early in its course. The condition is the most common soft tissue sarcoma in children. However, RMS in adults is an uncommon tumor that arises mainly in the large skeletal muscles (1-4). Pleomorphic RMS was first described by Stout in 1946 (5). More recent studies have reported that pleomorphic RMS is rare and occurs predominantly in adults. The present study describes a case of pleomorphic RMS in the right adrenal region of a 61-year-old female and reviews the literature on this rare disease. The study was approved by the ethics committee of the First Affiliated Hospital, School of Medicine, Zhejiang University (Hangzhou, China). Informed consent was obtained from the patient.

Correspondence to: Dr Jie Qin, Department of Urology, The First Affiliated Hospital, College of Medicine, Zhejiang University, 79 Qingchun Road, Hangzhou, Zhejiang 310003, P.R. China E-mail: qinjie@yeah.net

Key words: rhabdomyosarcoma, adrenal, adult

\section{Case report}

A 61-year-old female was referred to the urological ward of The First Affiliated Hospital, College of Medicine, Zhejiang University for a right adrenal mass that had been detected incidentally by ultrasound examination two weeks previously. The patient had no underlying disease and the physical examination was unremarkable. Abdominal ultrasound revealed a large adrenal tumor. A computed tomography scan revealed a right adrenal tumor measuring $6.0 \times 4.0 \mathrm{~cm}$ (Fig. 1). The patient had undergone a complete adrenal endocrinological evaluation, which demonstrated that the lesion was not a secreting tumor.

A pre-operative transcutaneous fine-needle aspiration biopsy was performed and the cytological diagnosis was consistent with a malignant neoplasm. Right adrenalectomy was performed. The tumor was observed to have invaded into the right lobe of the liver, and was well-demarcated from the hepatic parenchyma by a thick fibrous capsule. The total operating time was $3 \mathrm{~h}$. The estimated blood loss was $200 \mathrm{ml}$ (calculated and recorded by the attending anesthetist). The patient tolerated the procedure well and there were no post-operative complications. The drainage tube was removed at $48 \mathrm{~h}$ following the surgery. The patient was discharged on the fifth post-operative day, tolerating a regular diet. Pathological examination of the surgical specimen was pleomorphic RMS containing spindle cells (Fig. 2A). Immunohistochemistry revealed a positive stain for MyoD1, desmin, vimentin and CD56 (Fig. 2B-D). No expression of smooth muscle actin (SMA), SYN or S100 protein was identified in the tumor tissue.

\section{Discussion}

In the current World Health Organization Classification of Soft Tissue and Bone Neoplasms, RMS is divided into three distinct subtypes, embryonic, alveolar and pleomorphic (6). RMS is a rare disease of the adrenal gland neoplasm, which predominantly occurs in adults. Charytonowicz et al (7) suggest that RMS may arise from non-muscle cells, including mesenchymal stem cells. Theoretically, RMS may affect any body part, including the adrenal glands, as shown in the present case. To date, only two RMS cases in the adrenal region have 

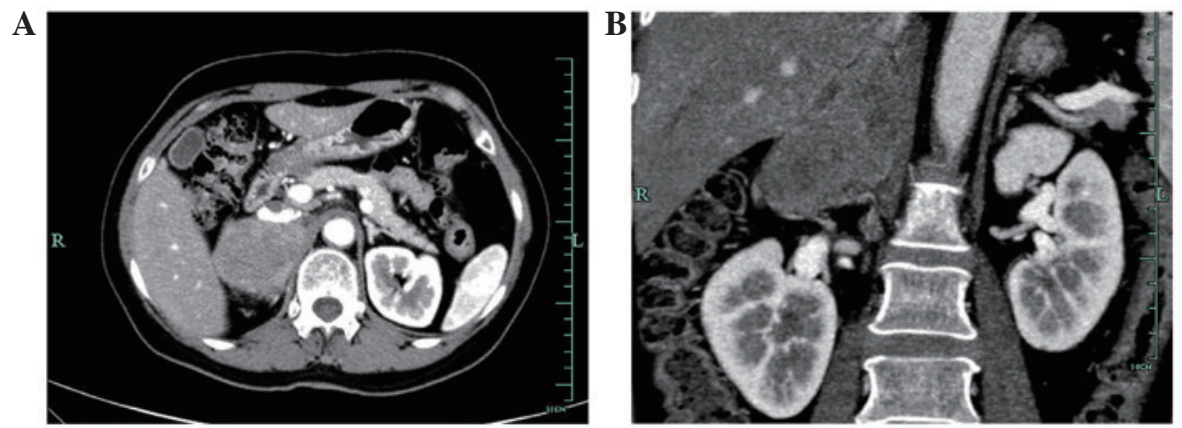

Figure 1. (A) Axial contrast-enhanced computed tomography (CT) scan showing the right adrenal tumor. (B) CT coronal section showing the right adrenal tumor.
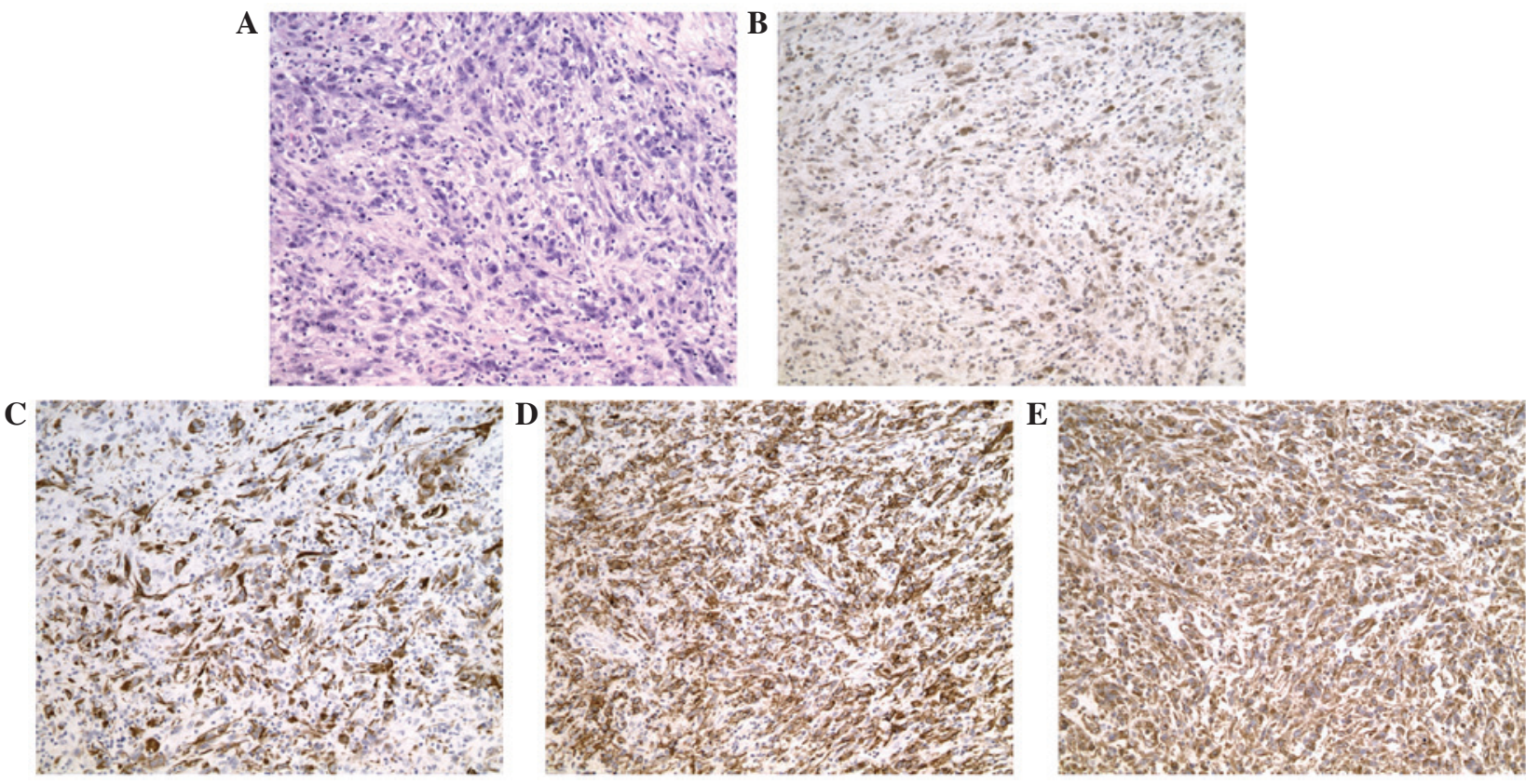

Figure 2. Pathological examination showing pleomorphic rhabdomyosarcoma. (A) Hematoxylin and eosin staining. Spindle-shaped cells arranged in a fascicular pattern were present. (B) Immunohistochemical staining showing positivity for (B) MyoD1, (C) desmin, (D) CD56 and (E) vimentin. Magnification, x200.

been described in the English literature. Yi et al (8) reported a case of alveolar RMS in the right adrenal region of a pediatric patient with a characteristic history of hypertension and fever. Katayama et al (9) reported a case of RMS in the adrenal region of an elderly hypertensive patient. However, pleomorphic RMS of the adrenal gland in an adult has not been previously reported.

In the present case, light microscopic examination revealed a malignant pleomorphic mesenchymal neoplasm, characterized mainly by the proliferation of atypical spindle cells and few epithelioid cells. Immunohistochemistry revealed positive staining for MyoD1, desmin, vimentin and CD56. By contrast, no expression of SMA, SYN or S100 protein was identified in the tumor tissue. A diagnosis of pleomorphic RMS was confirmed according to the clinical and pathological findings.

In conclusion, the present study described a rare case of pleomorphic RMS in the right adrenal region based on the histopathology and immunohistochemistry results. Due to the small number of described cases of adrenal gland RMS, inadequate information is available for evaluating the treatment procedure and the final prognosis of the patient. An accumulation of such cases and an improved understanding of the molecular biology driving RMS tumor behavior are required for further evaluation and research to identify the histogenesis of the condition. Primary pleomorphic RMS of the adrenal gland in an adult is a rare condition. To the best of our knowledge, this is the first case of pleomorphic RMS of the adrenal gland in an adult diagnosed by light microscopy and immunohistochemical staining.

\section{References}

1. Furlong MA, Mentzel T and Fanburg-Smith JC: Pleomorphic rhabdomyosarcoma in adults: a clinicopathologic study of 38 cases with emphasis on morphologic variants and recent skeletal muscle-specific markers. Mod Pathol 14: 595-603, 2001.

2. Ogilvie CM, Crawford EA, Slotcavage RL, et al: Treatment of adult rhabdomyosarcoma. Am J Clin Oncol 33: 128-131, 2010. 
3. Stock N, Chibon F, Binh MB, et al: Adult-type rhabdomyosarcoma: analysis of 57 cases with clinicopathologic description, identification of 3 morphologic patterns and prognosis. Am J Surg Pathol 33: 1850-1859, 2009.

4. Sultan I, Qaddoumi I, Yaser S, Rodriguez-Galindo C and Ferrari A: Comparing adult and pediatric rhabdomyosarcoma in the surveillance, epidemiology and end results program, 1973 to 2005: an analysis of 2,600 patients. J Clin Oncol 27: 3391-3397, 2009.

5. Stout AP: Rhabdomyosarcoma of the skeletal muscles. Ann Surg 123: 447-472, 1946.

6. Fletcher CDM, Unni KK and Mertens F (eds): Pathology and genetics of tumors of soft tissue and bone. In: Soft Tissue Tumors. IARC Press, Lyon, pp146-153, 2002.
7. Charytonowicz E, Cordon-Cardo C, Matushansky I and Ziman M: Alveolar rhabdomyosarcoma: is the cell of origin a mesenchymal stem cell? Cancer Lett 279: 126-136, 2009.

8. Yi X, Long X, Xiao D, Zai H and Li Y: Rhabdomyosarcoma in adrenal region of a child with hypertension and fever: A case report and literature review. J Pediatr Surg 48: e5-e8, 2013.

9. Katayama A, Otsuka F, Takeda M, et al: Rhabdomyosarcoma discovered in the adrenal region of an elderly hypertensive patient. Hypertens Res 34: 784-786, 2011. 